\title{
Construcción de paz desde las empresas: el papel de la RSE en contextos de postconflicto*
}

\section{Peacebuilding from companies: the role of CSR in post-conflict contexts}

\section{Resumen}

Los contextos de postconflicto demandan de diversos actores y acciones para construir la paz, resolver las consecuencias del conflicto y generar agendas para garantizar su no repetición. Entre estos actores se encuentran las empresas que, gracias a sus capacidades institucionales, pueden llevar a cabo una variedad de acciones y prácticas orientadas a la construcción de paz. En el presente artículo se desarrolla una reflexión teórica sobre las apuestas que las empresas pueden emprender desde la RSE en los contextos de postconflicto. Para ello, la reflexión presenta una revisión de la literatura académica sobre empresas y construcción de paz. Los resultados sugieren que las principales actividades que realizan las empresas desde la RSE son la vinculación de víctimas del conflicto y victimarios o excombatientes con la generación de empleos, implementación de modelos de ética empresarial y desarrollo de proyectos pedagógicos de emprendimiento y promoción de la paz. La discusión se enmarca en los roles directos e indirectos que asumen las empresas desde la RSE para la construcción de paz y el modo en el que estos se vinculan con pilares del contexto de postconflicto.

\section{Palabras clave}

postconflicto; paz; RSE; sostenibilidad.

\section{Abstract}

Post-conflict contexts demand various actors and actions to build peace, resolve the consequences of the conflict and generate agendas to ensure non-repetition. Among these actors are companies that, thanks to their institutional capacities, can carry out a variety of actions and practices aimed at building peace. This article develops a theoretical reflection on the bets that companies can undertake from CSR for post-conflict contexts. For this, the reflection presents a review of the academic literature on business and peacebuilding. The results suggest that the main activities that companies carry out from CSR are the linking of victims of the conflict and victimizers or ex-combatants with the generation of jobs, implementation of business ethics models and development of pedagogical projects for entrepreneurship and peace promotion. The discussion is framed in the direct and indirect roles assumed by companies from CSR for the construction of peace and how these are linked to pillars of the post-conflict context.

\section{Keywords}

post-conflict; peace; CSR; sustainability.

\footnotetext{
* El presente artículo de reflexión trae a colación algunas discusiones derivadas del proyecto de investigación denominado "Prácticas socialmente responsables de las organizaciones del Valle del Cauca y su aporte en la construcción de una Paz estable y duradera", financiado por la Fundación Universitaria Católica Lumen Gentium, Unicatólica y adscrito al Grupo Interdisciplinario de Investigación en Conocimiento Organizacional (GIICO) de la Facultad de Ciencias Empresariales.

** Administrador de Empresas y magíster en Psicología de la Universidad del Valle. Docente investigador, Grupo Interdisciplinario de Investigación en Conocimiento Organizacional (GIICO), Facultad de Ciencias Empresariales, Fundación Universitaria Católica Lumen Gentium, Unicatólica, Cali, Colombia. Correo electrónico: cbedoya@unicatolica.edu.co; https://orcid.org/0000-0001-9609-0319
} 


\section{Introducción}

Los contextos bélicos que se encaminan hacia escenarios de postconflicto han conllevado una serie de requerimientos y necesidades para construir la paz y asegurar la no repetición de fenómenos como la violencia y la guerra. Si bien esta labor está asociada a la responsabilidad de los gobiernos, algunas experiencias de países que estuvieron marcados por el conflicto evidencian el aporte de organismos internacionales, organizaciones del tercer sector y el sector empresarial para la construcción de paz (Forrer y Katsos, 2015; Grasa y Mateos, 2014; Kolk y Lenfant, 2013; Rettberg y Rivas, 2012).

Con relación al sector empresarial y productivo, diversas investigaciones han evidenciado el aporte que generan las empresas en la construcción de paz para escenarios de postconflicto (Forrer y Katsos, 2015; Kolk y Lenfant, 2013; Rettberg, 2002). De acuerdo con Ryan (2012), se considera que las empresas son fundamentales en la construcción de paz debido a que sus recursos, habilidades, mecanismos y operaciones contribuyen a resolver problemas derivados del conflicto y a consolidar la paz en las sociedades. Adicional a ello, las empresas pueden generar sinergia con los gobiernos para ampliar sus capacidades y de este modo, como actores de desarrollo económico y social, soportar procesos como los de reconciliación, resiliencia y memoria histórica (Gallego, Gutiérrez, Osorio, y Cortés, 2016).

Pese a que en la literatura académica se ha resaltado el papel de las empresas en la construcción de paz, algunas críticas y debates han cuestionado por qué las empresas deberían hacerlo, si las labores por construir la paz no constituyen actividades misionales propias de la generación del lucro (Prandi, 2010). Algunas respuestas a estas críticas se circunscriben bajo la noción de Responsabilidad Social Empresarial (RSE) que abarca tanto lo que las empresas hacen con sus ganancias, como también el modo en que lo hacen (Bond, 2014; Prandi, 2010).
Para Kytle y Ruggie (2005) la Responsabilidad Social Empresarial (RSE) va más allá de la filantropía y el cumplimiento que hagan las empresas del modo en que gestionan sus impactos económicos, sociales y ambientales, y sus relaciones con los grupos de intereses en todas sus esferas de influencia clave, como el lugar de trabajo, el mercado, la cadena de suministro, la comunidad y el ámbito de las políticas públicas. Expertos en materia de RSE como Archie Carroll sostienen que la justificación de los negocios para la implementación de la RSE obedece a cuatro argumentos: 1) la reducción de costos y riesgos; 2) el fortalecimiento de la legitimidad y de la reputación, 3) la construcción de una ventaja competitiva; y 4) la creación de situaciones gana-gana a través de la creación de valor sinérgico (Carroll y Shabana, 2010). Adicional a lo anterior, algunos académicos han sugerido como alternativa a la frase de Milton Friedman "el negocio del negocio es el negocio" que la responsabilidad social de las empresas es una vía para aumentar sus ganancias, "el negocio del negocio es la paz", la cual es buena para los negocios y la sociedad (Forrer, Fort y Gilpin, 2012).

Si bien se reconoce que la literatura que explora la relación empresa y paz se da partir de la década de 1990 (Bedoya-Dorado y Charfuelán, 2019a), es desde la década del 2000 -con el reporte The Business of Peace del entonces director del Foro Internacional de Líderes de Negocios (International Business Leaders Forum) (Nelson, 2000)-, que se asume una perspectiva sobre el modo en el que el sector privado puede participar en el avance de la agenda de la ONU para la construcción de paz, como se refleja en el Pacto Global y otras iniciativas (Bond, 2014). El mensaje principal de este reporte es que las empresas son capaces de aspirar más allá del cumplimiento de objetivos básicos normativos y el antiguo principio ético de "no hacer daño" (Nelson, 2000).

Posterior a este periodo se encuentran revisiones en la literatura que exploran otros 
aspectos relacionados con los negocios y la paz (Bedoya-Dorado y Charfuelán-Aguirre, 2019a; Fort y Schipani, 2002; Oetzel, Getz, y Ladek, 2007; Oetzel, Westermann-Behaylo, Koerber, Timothy y Rivera, 2009). Por una parte, algunas investigaciones registran diversas prácticas de las empresas que se pueden orientar hacia el desarrollo económico mediante la generación de empleos y propuestas de emprendimiento, la adopción de principios de evaluación externa; la contribución de un sentido de comunidad; el involucramiento con la cooperación y la diplomacia; y la participación de la evaluación del riesgo de conflictos (Banks, 2016; Oetzel et al., 2009; van Dorp, 2017; Yoosuf y Premaratne, 2017).

Teniendo en cuenta que la RSE le permite a las empresas articularse con la paz, el objetivo del presente artículo es analizar cuáles son las prácticas de RSE orientadas a la construcción de paz en contextos de postconflicto. Esta discusión se enmarca en los aportes teóricos y empíricos registrados en una revisión de la literatura derivada del proyecto de investigación denominado "Prácticas Socialmente Responsables de las organizaciones del Valle del Cauca y su aporte en la construcción de una Paz estable y duradera" (Bedoya-Dorado y Charfuelán-Aguirre, 2019a; 2019b). Las prácticas de RSE registradas en los artículos y libros que componen la literatura seleccionada se agruparon y analizaron bajo el modelo de Hamre y Sullivan (2002) sobre los pilares en el postconflicto y la pirámide de Lederach -adaptada por Killick (Nelson, 2000)- sobre los roles de las personas en los negocios y los roles directos e indirectos de las empresas para la construcción de la paz.

Las reflexiones que suponen la relación contexto-empresa son de gran importancia para países como Colombia que desde el 2016 ha estado inmerso en un contexto de postconflicto producto de la firma del Acuerdo de la Paz entre el Gobierno Nacional y las FARC-EP (Gobierno Nacional de Colombia y FARC-EP, 2016). Pese a que en la literatura existen publicaciones centradas en el contexto colombiano, la mayoría de estas se orientan a la construcción de paz en el escenario de conflicto (Jiménez-Peña, 2014; Miklian y Rettberg, 2017; Rettberg, 2002; 2007; Rettberg, Leiteritz, y Nasi, 2011; Rettberg y Rivas, 2012; Ruiz, 2003; Vargas, 2012), las que se centran en un contexto de postconflicto o postacuerdo son escasas (Barrios, 2016; Bedoya-Dorado y Charfuelán, 2019b; González, 2016; Rodríguez y Gamboa, 2017). Adicionalmente, esta reflexión brinda alguna claridad y señala uno de los modos en los que las empresas pueden actuar y aportar prácticas para la construcción de paz desde su quehacer.

El artículo se estructura en tres partes: primero se presenta una aproximación teórica a la RSE; seguido, se exponen elementos conceptuales sobre la construcción de paz en contextos de postconflicto y la discusión sobre la construcción de paz desde la RSE; y finalmente, se despliegan las conclusiones de la discusión y recomendaciones para las empresas que deseen implementar o desarrollar este tipo de apuestas.

\section{Aproximación a las prácticas de Responsabilidad Social Empresarial}

Con respecto a la aplicación al campo empresarial, la RSE tiene sus orígenes a mediados del siglo XX con la publicación de Howard Bown, Social Responsabilities of the Businessman en 1953. Para entonces, este autor asociaba la RSE a la obligación de seguir las políticas y tomar decisiones que fuesen deseables en términos de objetivos y valores de la sociedad (Carroll, 1999; Melo, Sánchez y García, 2011). Otras aproximaciones como las de Freeman (1984) limitaban la actuación de la RSE al ejercicio de maximizar las ganancias para beneficiar a los dueños del capital y al Estado de manera simultánea.

El discurso estratégico posterior a la década de 1960 permeó la noción de RSE, la propuso como una fuente de ventaja competitiva, oportunidades e innovación, que además de servir como estrategia 
defensiva ante acusaciones y demandas de la opinión pública, servía como estrategia para crear valor (Fuentes-García, Núñez-Tabales, y Veroz-Herradón, 2008; Porter y Kramer, 2002; 2011). Así mismo, la consideración de múltiples actores en la actividad empresarial (sociedad, medioambiente, Estado, accionistas, clientes, trabajadores, etc.) abordó la RSE como enfoque de gestión estratégico que se puede integrar a toda la organización y se orienta a suplir y satisfacer las necesidades y expectativas de dichos grupos de interés (stakeholders) (Dentchev, 2004; Porter y Kramer, 2002; 2011; Raufflet, 2010)

La inclusión de la noción de grupos de interés - "colectivos o entidades que están o pueden verse afectadas por los servicios o actividades de una empresa y aquellas otras cuyas opiniones o decisiones afectan o pueden afectar a los resultados económicos o la reputación de la misma" (Ibisate, 2008, p.127)-, permite esclarecer múltiples relaciones y además, implica para las organizaciones evaluar el modo en el que estos actores se ven afectados y el modo en que se ha de atender dichas afectaciones. Esta noción parte del enfoque de "esferas de influencia empresarial", con el cual se determina el contexto próximo del marco de acción de las empresas y el nivel de influencia de sus grupos de interés (Melo et al., 2011).

La revisión de la literatura académica sugiere que existen múltiples definiciones de RSE (Dahlsrud, 2008; Rahman, 2011; van Marrewijk, 2003), algunas de ellas han sido predominantes por el modo consensuado de su construcción y por su aplicación de manera estandarizada a través de guías como la ISO 26.000. De acuerdo con esta guía, la RSE es:

La responsabilidad de una organización ante los impactos de sus decisiones y actividades en la sociedad y el medio ambiente, a través de un comportamiento transparente y ético que sea: consistente con el desarrollo sostenible y el bienestar general de la sociedad; considere las expectativas de sus partes interesadas; esté en cumplimiento con la legislación aplicable y sea consistente con normas internacionales de comportamiento; y esté integrada a través de toda la organización. (Organización Internacional de Normalización, 2014)

Otras nociones han ampliado el espectro de la RSE para proponer que esta no se limita a la filantropía, donaciones o acciones comunitarias, sino que es un imperativo en el contexto regional, dadas las condiciones sociales, es una estrategia integral del negocio, es decir, de toda la organización. Como iniciativa, la RSE fortalece las capacidades de las organizaciones y promueve el desarrollo del contexto en el cual opera, implica un compromiso voluntario y la incorporación de valores éticos en la toma de decisiones y en la relación con sus grupos de interés; es una oportunidad para la construcción de un espacio de diálogo y articulación entre los diversos actores sociales de las comunidades o de la sociedad, y es un ejercicio de construcción de confianza. Así mismo, la RSE trasciende el mero cumplimiento del marco normativo y se compromete con la dimensión ambiental, económica y social del contexto en el cual están inmersas las empresas y en el que tienen impactos (Melo et al., 2011).

Garriga y Melé (2004) clasificaron las teorías que permiten comprender el quehacer de la RSE bajo cuatro enfoques: teorías instrumentales, teorías políticas, teorías integrativas y teorías éticas. En el enfoque instrumental la RSE es vista solo como una herramienta estratégica para lograr objetivos económicos y generar riqueza; esto se da porque la RSE permite mejorar el desempeño organizacional y el valor para los accionistas. Los fundamentos teóricos de esta perspectiva instrumental derivan principalmente de enfoques económicos como el neoclásico, bajo el cual los propietarios de una empresa esperan que sus gerentes y trabajadores persigan las metas de desempeño financiero a cambio de una remuneración justa y otro tipo de beneficios que otorgan un contrato de trabajo (Garriga y Melé, 2004; Voegtlin y Greenwood, 2016). 
En el segundo enfoque las teorías se concentran en las interacciones y conexiones entre negocios y sociedad, y en el poder de las empresas en la sociedad y las posiciones de los empleados con respecto a las empresas, lo cual incluye consideraciones políticas y un análisis político en el debate del quehacer de la RSE. Con esta aproximación la empresa es considerada como un actor económico y a la vez político, además de su poder económico para alcanzar el lucro, hace parte de la "ciudadanía" de una sociedad y gracias a su poder e influencia, asume responsabilidades políticas, especialmente cuando el Estado no puede o no quiere hacerlo (Garriga y Melé, 2004; Voegtlin y Greenwood, 2016).

Este enfoque político es ampliamente discutido en la literatura académica, en la que la naturaleza del rol político de las empresas en la sociedad hace alusión a la provisión de bienes públicos y la contribución a la autorregulación de los negocios. Voegtlin y Greewood (2016) encuentran que este enfoque ha cobrado un impulso significativo durante los últimos años, principalmente para dar respuesta a los desafíos de la globalización, se preocupa por los derechos y responsabilidad de los diferentes grupos de interés, y las implicaciones de las responsabilidades políticas de una organización dentro de una sociedad y a través de las sociedades (Baek y Kim, 2014; Garriga y Melé, 2004).

Otra de las perspectivas de la RSE es el enfoque social integrativo o teorias integrativas, se fundamentan en la teoría de los grupos de interés y de valor compartido (Freeman, 1984; Porter y Kramer, 2011). Este grupo de teorías se concentran en la manera en la que las empresas integran demandas sociales al argumentar que estas dependen de la sociedad para su existencia, continuidad y crecimiento. Bajo esta perspectiva se considera que las demandas sociales son la ruta mediante la cual las sociedades interactúan con las empresas y les otorgan cierta legitimidad y prestigio. El supuesto de esta fundamentación es que la creación de valor para los grupos de interés es el pilar de las empresas, las cuales operan en un contexto capitalista. Ello implica que los intereses de los grupos de interés se deben atender con el objetivo de crear situaciones gana-gana para los negocios y la sociedad (Garriga y Melé, 2004).

Finalmente, las teorías éticas se centran en los requerimientos éticos para cimentar las relaciones entre las empresas y la sociedad. Estas se basan en principios que expresan el modo correcto de hacer las cosas o lo necesario para alcanzar una "buena sociedad" (Garriga y Melé, 2004). Noland y Phillips (2010) argumentan que las preocupaciones éticas pueden formar la base para examinar las estrategias organizacionales y hacer que estas se vuelvan estratégicamente relevantes, ya que el objetivo final es la creación de valor a largo plazo.

Los distintos enfoques para comprender la RSE presentan un común denominador: el involucramiento de las empresas con actores internos y externos que constituyen sus grupos de interés. Así mismo, el quehacer e impacto de la RSE va más allá de los objetivos organizacionales, trasciende a las sociedades puesto que, como señala el Consejo Empresarial Mundial para el Desarrollo Sostenible (World Business Council for Sustainable Development), la RSE supone un compromiso continuo por parte de las empresas a comportarse éticamente y contribuir al desarrollo económico, al tiempo que mejoran la calidad de vida de la fuerza laboral y sus familias, así como de la comunidad local y la sociedad en general (Wilcox, 2006).

\section{Prácticas de construcción de paz desde la RSE en contextos de postconflicto}

\subsection{La construcción de paz en el postconflicto}

La noción de "construcción de paz" o peacebuilding tiene sus orígenes en los esfuerzos de las Naciones Unidas una vez finalizó la Guerra Fría, y el despliegue de operaciones de paz para concluir 
con los conflictos que afectaban a distintos países del mundo (Grasa y Mateos, 2014). Las lecciones aprendidas en los contextos bélicos hicieron cambiar el modo en el que se construye la paz, por lo que las acciones encaminadas a la culminación de la violencia armada de corto plazo han dado paso a estrategias de largo plazo, orientadas a identificar las causas de los conflictos, actuar sobre las crisis humanitarias y hacer sostenibles las acciones de paz a través de los aportes de múltiples actores (Grasa y Mateos, 2014).

De acuerdo con lo anterior, es posible distinguir dos orientaciones en el marco de la construcción de paz: por una parte, el peacemaking que hace alusión a los procesos de establecimiento de paz, en el que las acciones buscan un acuerdo entre las partes que representan los actores del conflicto con alternativas pacíficas. Por otra, el peacekeeping que se refiere al mantenimiento de la paz y la prevención de la recaída en el conflicto (Boutros-Ghali, 1992). Esta distinción permite señalar que el peacemaking se da en los contextos de conflicto, mientras que el paecekeeping ocurre en los de postconflicto y que los esfuerzos para la construcción de paz en cada uno de ellos son diferentes y específicos.

Los contextos de postconflicto constituyen un escenario de transición de la violencia hacia la paz, a partir de acuerdos y negociaciones para finalizar con el conflicto u otro tipo de formas, como las victorias militares. Este escenario se convierte en una novedad para los países o regiones en los que se presenta y derivan una serie de desafíos, como las políticas para el sostenimiento de la paz, las violencias postconflicto, los procesos de justicia transicional, la reintegración, la reparación, la reconciliación, la cultura para la paz, etc. (Barnett, et al., 2007; Flavin, 2003; Rathmell, 2005; Ugarriza, 2013; Wielandt, 2005).

Para Ugarriza (2013), el postconflicto es una solución del conflicto que se ubica en un momento temporal y en el que se propicia el fenómeno de construcción de paz, como fortalecimiento y soporte para solidificar la paz y evitar la recaída en el conflicto. Este contexto permite la inserción de excombatientes, dejación de armas, remoción de minas antipersonas, repatriación de refugiados, protección de los derechos humanos, cuidado del medio ambiente, fortalecimiento y cambios de las instituciones gubernamentales, apoyo en la reconciliación de la sociedad, procesos de participación ciudadana, entre otros más (Rettberg et al., 2002). No obstante, autores como Flavin (2003) precisan que el postconflicto sugiere una terminación de un conflicto, pero no su resolución, la cual es un proceso a largo plazo.

La definición utilizada por el Banco Mundial desde 1995 señala que las actividades en el postconflicto se enfocan en la necesidad de reconstruir un marco socioeconómico para la sociedad y la reconstrucción de condiciones para el funcionamiento de una sociedad en paz, lo que incluye un marco de gobernanza y las reglas de las leyes (World Bank, 1995). De acuerdo con Hamre y Sullivan (2002), existen cuatro pilares en el postconflicto sobre los cuales se debe actuar:

1. Seguridad. Hace alusión a todos los aspectos de seguridad pública, en particular, a la creación de un ambiente de seguridad y el desarrollo de instituciones de seguridad legítimas y efectivas.

2. Justicia y reconciliación. Todas las necesidades para tratar con abusos del pasado a través de mecanismos formales e informales para resolver quejas que surgen del conflicto y crear un sistema legal imparcial y responsable para el futuro, en particular, mediante la construcción de un aparato efectivo de aplicación de la ley, un sistema judicial abierto, leyes justas, y un sistema de correcciones humanas. 
3. Bienestar social y económico. Orientado a las necesidades sociales y económicas en particular, que brinden ayuda de emergencia, restauración de servicios como los de salud y educación, establecimiento de las bases para una economía viable y programas de desarrollo sostenible e inclusivo.

4. Gobernanza y participación. Aborda la necesidad de crear instituciones políticas y administrativas legítimas y efectivas, así como procesos participativos para fortalecer la gestión del sector público y garantizar la participación activa y abierta de la sociedad civil en la formulación del gobierno del país y sus políticas.

\subsection{La RSE y su articulación con la construcción de paz}

Comose hamencionado, sibien la construcción de paz es una necesidad y preocupación de los gobiernos principalmente, las empresas pueden contribuir a soportar sus actividades (Forrer y Katsos, 2015; Kolk y Lenfant, 2013; Rettberg, 2002; Rettberg y Rivas, 2012) y según la literatura, las prácticas de RSE se articulan como uno vehículo para este fin (Bond, 2014; Fort, 2007; Fort y Schipani, 2002; McKenna, 2014; Oetzel et al., 2007; Oetzel et al., 2009; Prandi, 2010).

Para comprender el modo en que las empresas contribuyen a la construcción de paz desde la RSE es importante tener en cuenta dos niveles de análisis; por una parte, las acciones directas para la construcción de paz que se relacionan con el enfoque social integrativo o teorías integrativas de la RSE (Freeman, 1984; Garriga y Melé, 2004; Porter y Kramer, 2011), en las que las empresas asumen la construcción de paz como una demanda social e identifican grupos de intereses con el fin de crear valor para ellos (McKenna, 2014; Prandi, 2010).
Por otra parte, las acciones indirectas basadas en las teorías éticas de la RSE (Garriga y Melé, 2004; McKenna, 2014; Noland y Phillips, 2010), en la que las acciones éticas de los negocios promueven la mejora de condiciones sociales y económicas que contribuyen a la paz, sin la necesidad de transformar el gobierno corporativo o crear programas o prácticas de RSE específicos para la paz (Forrer et al., 2012; Fort, 2007; McKenna, 2014; Oetzel et al., 2009).

\subsubsection{Acciones directas de RSE en la construcción de paz}

De acuerdo con Prandi (2010), en contextos de postconflicto es necesario incorporar criterios de lucha contra la pobreza y de reconstrucción física en las políticas de RSE, así como divisiones sociales desde una perspectiva de transparencia y equidad: "una política de relación con los stakeholders que incorpore una perspectiva de construcción de paz debe ser clave para garantizar una correcta inserción de la empresa en contextos de postconflicto" (p. 39).

Para Bennett (2002), Rodríguez y Gamboa (2017), Prandi (2010) y Wilson (2015), las empresas deben prestar una atención especial para determinar la presencia y su relación con grupos como víctimas (individuales y colectivas) de violaciones de derechos humanos; desplazados, refugiados y retornados; desmovilizados y excombatientes; minorías y poblaciones históricamente discriminadas, y miembros de las fuerzas armadas y empresas de seguridad privadas, teniendo en cuenta variables como las de género, edad, raza, tipo de población, situación política y económica, etc.

Grupos como los desmovilizados o excombatientes, debido al daño ocasionado en la convivencia social, son excluidos socialmente o marginalizados por las poblaciones civiles y presentan condiciones de desigualdad; como por ejemplo, en habilidades y competencias para el trabajo (Hamre y Sullivan, 2002; Mckenna, 2014; Rathmell, 2005; 
Rolston, 2007; Vargas, 2012). Rolston (2007) considera que entre los mismos excombatientes es posible identificar grupos vulnerables, como el caso de las mujeres cuando se comparan con los hombres; ellas tuvieron experiencias específicas en el marco de los conflictos, este es el caso de los embarazos y la maternidad. Además, son excluidas de asociaciones de militares retirados y presentan condiciones de inequidad con relación a beneficios de pago, distribución de tierras y entrenamientos.

Para las empresas resulta ser de gran importancia reconocer tanto la presencia como la historia de los diferentes grupos en el conflicto, en algunos casos estos podrían ser empleados, proveedores, socios, comunidades cercanas a las áreas de operaciones de las empresas, o sus clientes potenciales (Bennett, 2002; Rodríguez y Gamboa, 2017; Prandi, 2010; Wilson, 2015).

Algunas investigaciones sugieren que las prácticas de RSE tienen impacto social y económico en los contextos de postconflicto (Barrios, 2016; García, Durán, Parra y Martínez, 2018; Ledbetter, 2016; Maconachie, 2016; Rathmell, 2005; Rolston, 2007; Sepúlveda et al., 2015). Este es el caso de la generación de empleos bajo un enfoque inclusivo, en el que se reclutan poblaciones víctimas del conflicto y desmovilizados para conformar el personal de las empresas. Este tipo de apuestas no solo buscan contribuir a superar la vulnerabilidad y las condiciones de pobreza de algunos de estos actores, sino que también actúan como procesos de atención y reparación de víctimas, fortalecimiento de una cultura de paz, contribución a la inserción a la vida civil, procesos de reconciliación, y promoción de la convivencia en la sociedad.

Para Prandi (2010) las empresas también pueden contribuir al aumento de los ingresos per cápita de las economías, a través de la compra de materias primas, bienes y servicios, y la creación de oportunidades de trabajo y de emprendimiento.
Es por ello que es importante reconocer las actividades económicas locales y apoyar sus capacidades. No obstante, la generación de empleos debe ser sostenible y de calidad, con salarios que satisfagan las necesidades básicas de los empleados y sus familias. Para la OIT, el trabajo decente es la principal vía para salir de la pobreza, fenómeno característico de más de la mitad de la población de los países en vía de desarrollo y economías en transición (Prandi, 2010).

La generación de empleos y la contratación de los grupos de interés desde una perspectiva de construcción de paz implica políticas de contratación diferentes a los procesos tradicionales de recursos humanos, bajo elementos no discriminatorios (e.g. de etnias o grupos sociales) y con el reconocimiento de los niveles bajos de formación de las poblaciones (Prandi, 2010).

Adicional a ello, las investigaciones señalan que las condiciones de pobreza y vulnerabilidad hacen que las comunidades sean más susceptibles a iniciar actividades al margen de la ley y reproducir círculos de violencia (Barnett et al., 2007; Chowdhury, 2011; Kanashiro y Starik, 2016; Oetzel et al., 2009; Penh, 2009), que a su vez afectan el quehacer de las empresas (Forrer y Katsos, 2015; Gallego et al., 2016; Oetzel y Breslauer, 2015; Oetzel et al., 2007; 2009; Prandi, 2010; Rettberg, 2007). Esto se sustenta además en estudios que evidencian que las economías inmersas en escenarios de conflicto tienden a disminuir su PIB (Ruiz et al., 2017).

Algunas investigaciones sugieren que complementario a la generación de empleos, otras prácticas de RSE orientadas a superar las condiciones de pobreza y vulnerabilidad son el apoyo a la creación y fortalecimiento de emprendimientos, las cuales requieren además de proyectos pedagógicos orientados a la formación de competencias y habilidades para el trabajo, proyectos productivos y fortalecimiento de los conocimientos de cada uno 
de los actores (Banks, 2016; Jiménez-Peña, 2014; Ramnarain, 2015). Algunas empresas capacitan en planes de negocios a las personas interesadas en crear su propia empresa, de modo que estos puedan vincularse a ellas como proveedores de productos o servicios cuando cumplan con requisitos de calidad (Prandi, 2010).

Estos proyectos, al igual que los empleos inclusivos, reconocen que entre los actores del postconflicto se encuentran segmentos más vulnerables que han sido excluidos y marginalizados socialmente o que de manera particular, sobrellevaron otros efectos del conflicto violento, como es el caso de las mujeres, jóvenes, adultos mayores y reinsertados, por lo cual las capacitaciones y aprendizajes deben asumir una postura de equidad y oportunidad (Banks, 2016; Chowdhury, 2011; Jiménez-Peña, 2014; Kanashiro y Starik, 2016; Penh, 2009; Rettberg y Rivas, 2012).

Los proyectos pedagógicos no solo se encuentran registrados en las investigaciones como prácticas de RSE para el emprendimiento y desarrollo económico, sino que además existen otros orientados al fortalecimiento de una cultura de paz y la contribución a un sentido de comunidad, en el que aparecen intervenciones y programas para la paz y la no violencia, la formación de derechos humanos y equidad de género, prevención de violencia de género y otras formas de violencia, patrocinios y becas escolares, desarrollo de actividades deportivas para la participación de las comunidades y promoción de valores universales, etc. (De-San-Eugenio et al., 2017; Fort y Schipani, 2007; Hagerty, 2013; Hayhurst y Szto, 2016; Jiménez-Peña, 2014; Oetzel et al., 2009; van Dorp, 2017; Wilson, 2015).

Debido a que la violencia aconteció en territorios o áreas geográficas específicas, sus efectos tienen un impacto directo en el medio ambiente y la naturaleza (Bedoya, 2017; Hönke, 2014; Mckenna, 2014, 2016; Molina, 2004; Prandi y Lozano, 2010), lo que a su vez afecta las condicio- nes de vida de las poblaciones y especies vegetales y animales. Este es el caso de la contaminación de los recursos hídricos, la transformación de los ecosistemas y la destrucción de las tierras para las actividades agrícolas (Jiménez-Bautista, 2012; Prandi y Lozano, 2010). No obstante, la literatura académica es escasa sobre las prácticas de RSE orientadas a la reparación del daño del medio ambiente y su protección, esta dimensión solo se involucra cuando se hace mención a los derechos humanos, con la idea de aumentar la consciencia de conservar el medio ambiente (Buitrago et al., 2015; Mckenna, 2014, 2016; Prandi y Lozano, 2010).

Investigaciones como las realizadas en Rwanda encuentran prácticas de creación de paz desde la apuesta del turismo, en las que se tiene en cuenta el respeto por el medio ambiente y su conservación (Boudreaux, 2007; Prandi y Lozano, 2010; Tobias y Boudreaux, 2011). Otro caso es el de la empresa Shell en Nigeria, la cual se comprometió con la reducción de los impactos negativos sobre el medio ambiente a través de proyectos para terminar con la quema de gas y la protección de la biodiversidad en el delta (Prandi y Lozano, 2010).

Finalmente, las investigaciones señalan que con el objetivo de aumentar el impacto de las prácticas de RSE en los contextos de postconflicto, las empresas deben unir sus esfuerzos con otro tipo de organizaciones tanto del mismo sector productivo, como entidades públicas, organismos nacionales e internacionales, y organizaciones del tercer sector. Esto permite generar una sinergia en la que las capacidades institucionales de las empresas soportan la experiencia y conocimiento que poseen las demás organizaciones para desarrollar apuestas o prácticas para la construcción de paz (Abramov, 2009; Ford, 2015; Gerson, 2001; Ghimire y Raj Upreti, 2012; Jiménez-Peña, 2014; Katsos y Forrer, 2014; Mckenna, 2016). Así mismo, las experiencias en contextos de postconflicto indican que la combinación de financiación y know how de los diversos actores es lo más indicado para los fines de la paz (Prandi, 2010). 
De acuerdo con Prandi (2010), ningún actor u organización está en la capacidad de afrontar por si solo los desafíos que suponen los contextos de postconflicto, como es el caso de reconstrucción social, política y económica de un país, por lo cual la clave está en la articulación coordinada y eficaz de las capacidades de cada uno de los actores implicados. Es por ello que las empresas deben incluir las alianzas con otros actores en sus políticas de RSE con el objetivo de conseguir de manera efectiva los objetivos de construcción de paz. Esto implica considerar que las empresas son un actor más en un entramado complejo de relaciones entre actores que buscan construir paz y su actuar se puede desarrollar, no solo mediante diversas prácticas de RSE, sino también desde diferentes niveles. En la siguiente figura se presenta la adaptación que realiza Killick (Nelson, 2000) a la pirámide de Lederach sobre los actores y enfoques para la construcción de paz, la cual se concentra en roles de las personas en los negocios:
TIPO DE ACTOR

Liderazgo superior

- CEO/ Junta directiva/presidente

- Política a nivel corporativo y función

de asuntos públicos

Liderazgo de rango medio

- Gerentes regionales/gerentes nacionales de transnacionales

- CEO de la compañía nacional

Liderazgo de base

- Gerentes de unidades de negocios

- Expertos funcionales (e.g.

Gerente de RSE

- Gerentes de pymes

ROLES/ACTIVIDADES

Compromiso con gobiernos nacionales, agencias intergubernamentales, ONG internacionales y otras compañías.

Compromisos con temas de reformas del sector público, impuestos, corrupción, leyes del sector privado, bancos, derechos humanos, democratización, etc.

Diálogo político regional

\section{Gestión de los impactos} y relaciones a nivel local
Derechos de mujeres, consultas a comunidades, subcontratantes, desarrollo empresarial, ONG, construcción de capacidades, etc.

Figura 1. Los roles para las personas en los negocios para la paz Fuente: adaptación de Bond (2014)

La figura 1 muestra un sistema de clasificación para el ambiente de los negocios en el que se presentan los niveles en los que las personas, especialmente líderes, pueden gestionar dimensiones del postconflicto. Esto permite comprender que en las sociedades, organizaciones y grupos existen jerarquías de responsabilidades y de autoridad. Así mismo, las personas están mediadas por sus niveles en los negocios y esferas de influencia para actuar sobre las problemáticas del postconflicto y promover la paz.

\subsubsection{Acciones indirectas de RSE para la construcción de paz}

Autores como Forrer et al. (2012), argumentan que no es que las empresas deban promover la paz, sino que dado que poseen atributos no violentos en sus acciones y sus acciones son éticas, estas ya están contribuyendo a la paz y conocer las posibles consecuencias de ello otorga una motivación adicional para continuar siendo ético. McKenna (2014) considera el anterior como el rol indirecto 
que desempeñan las empresas en la construcción de paz a través de los negocios, como algo "usual" en su quehacer. Esto quiere decir que las empresas no deben cambiar sus procesos misionales para orientarse hacia la paz, sino que sus actividades productivas pueden mejorar condiciones sociales y económicas que contribuyen y promueven la paz. Adicional a ello, Fort (2007) argumenta que las contribuciones empresariales para una paz sostenible no requieren una transformación del gobierno corporativo, sino un enfoque más fuerte para las comprensiones comúnmente aceptadas de las prácticas éticas de los negocios.

Las estrategias que enfatizan en las formas indirectas para la construcción de paz desde las empresas son principalmente pragmáticas y atractivas para ellas (McKenna, 2014, Oetzel et al., 2009). Cuando algunas empresas consideran la construcción de paz desde una perspectiva corporativa la vinculan desde la RSE, sin que se llame propiamente "construcción de paz", por ser un término demasiado político. Adicional a ello, existe la creencia que las empresas no tienen la experiencia ni la capacidad para adoptar un rol proactivo en esta actividad. Es por ello que algunas empresas, en lugar de generar nuevas políticas y programas bajo la etiqueta de "construcción de paz", aportan a la paz bajo las prácticas de RSE existentes. Este fenómeno sugiere que el rol de las empresas en la construcción de paz no solo es indirecto, sino también voluntario (Fort, 2007; McKenna, 2014).

Las investigaciones evidencian que a través de la RSE las empresas pueden implementar estrategias de reconstrucción posteriores al conflicto, que abordan algunas de sus causas como la corrupción, por ejemplo. Este fenómeno se presenta tanto en los ámbitos de los gobiernos, como también en ejercicios empresariales propios del sector productivo (Abramov, 2009; Barnett et al., 2007; Bond, 2014; Forrer y Katsos, 2015; Montinola y Jackman, 2002; Oetzel et al., 2009). Para autores como Oetzel et al. (2009) existe una correlación positiva en- tre los altos niveles de corrupción y los conflictos violentos de países. En estos casos, las empresas pueden trabajar con otro tipo de organismos para limitar la corrupción en su esfera de influencia y apoyar esfuerzos para reducir la corrupción y hacer cumplir la ley contra sobornos. Estas contribuciones pueden tener un impacto sustancial en el ambiente general de los negocios y la promoción de la paz.

Para estos autores, uno de los principales retos asociados a la reducción de la corrupción y el incremento de la transparencia es la acción colectiva que se requiere para crear cambio. Al combatir la corrupción, las empresas cumplen con las leyes internacionales. Este es el caso de la Convención de la Organización para la Cooperación y el Desarrollo Económicos (OCDE) sobre la lucha contra el soborno de funcionarios públicos en transacciones comerciales, conocida como la Convención contra el soborno de la OCDE, la cual obliga a sus Estados miembros a penalizar el soborno de funcionarios públicos en la gestión de negocios internacionales (Bennet, 2002).

La lucha contra la corrupción se asocia con el actuar ético de la RSE (Garriga y Melé, 2004), el cual puede incorporar modelos de ética empresarial y códigos de ética para los empleados, que se consideran una contraprestación ética y moral a la sociedad por permitir su accionar (Abramov, 2009; Jiménez-Peña, 2014; Wilcox, 2006). Para materializar estas acciones, existen algunas iniciativas como las de adoptar códigos internacionales de conducta relacionados con las prácticas laborales y el actuar en la sociedad, por ejemplo, los principios rectores de la ONU en materia de derechos humanos, adherirse al Pacto Global de las Naciones Unidas y al cumplimiento de los Objetivos de Desarrollo Sustentable (ODS), el uso de la guía de la ISO 26.000 y de la Organización para la Cooperación y el Desarrollo Económico (OCDE), la iniciativa de reportes, etc. (Oetzel et al., 2009; Prandi y Lozano, 2010; Rettberg y Rivas, 2012). Estas iniciativas, 
además de contribuir a la lucha contra la corrupción, promueven el rechazo de economías ilegales y el respeto por los derechos humanos (Buitrago et al., 2015; Fort y Schipani, 2002; McNulty, 2014).

En materia de derechos humanos, Prandi (2010) argumenta que estos imponen una serie de derechos y obligaciones para las empresas en materia de personal y activos e inversiones de capital. Entre algunas restricciones de estos derechos, se encuentra el trabajo forzoso, el cual, según la OIT, incluye dos elementos: la prestación de un trabajo bajo amenaza y su realización no voluntaria. En Latinoamérica y el Caribe existen cerca de un millón y medio de víctimas de trabajo forzoso, de las cuales el $75 \%$ son el resultado de la explotación económica de actores privados.

Finalmente, adherirse al Pacto Global y la consecución de los ODS se articulan como prácticas de RSE que tienen incidencia en la construcción de paz.
Entre estos objetivos es posible destacar: paz, justicia e instituciones sólidas; fin de la pobreza; educación de calidad; trabajo decente y crecimiento económico; reducción de las desigualdades, etc. De acuerdo con Allan (2001), estos compromisos posibilitan crear condiciones de calidad de vida para todas las generaciones y aportar al desarrollo y la reconstrucción de los países que buscan construir la paz.

En la figura 2 se presentan -a manera de resumen- las prácticas de RSE que pueden realizar las empresas para la construcción de paz en los contextos de postconflicto. De acuerdo con la revisión de la literatura, estas prácticas constituyen en su mayoría acciones directas de las empresas para la construcción de paz, y se orientan a los pilares de bienestar social y económico, justicia y reconciliación, y gobernanza y participación. Además, de acuerdo con lo expuesto en la figura 1 , estas prácticas se pueden gestionar a partir de diversos niveles del rol en los negocios, en los cuales destaca principalmente el papel de liderazgo de base.

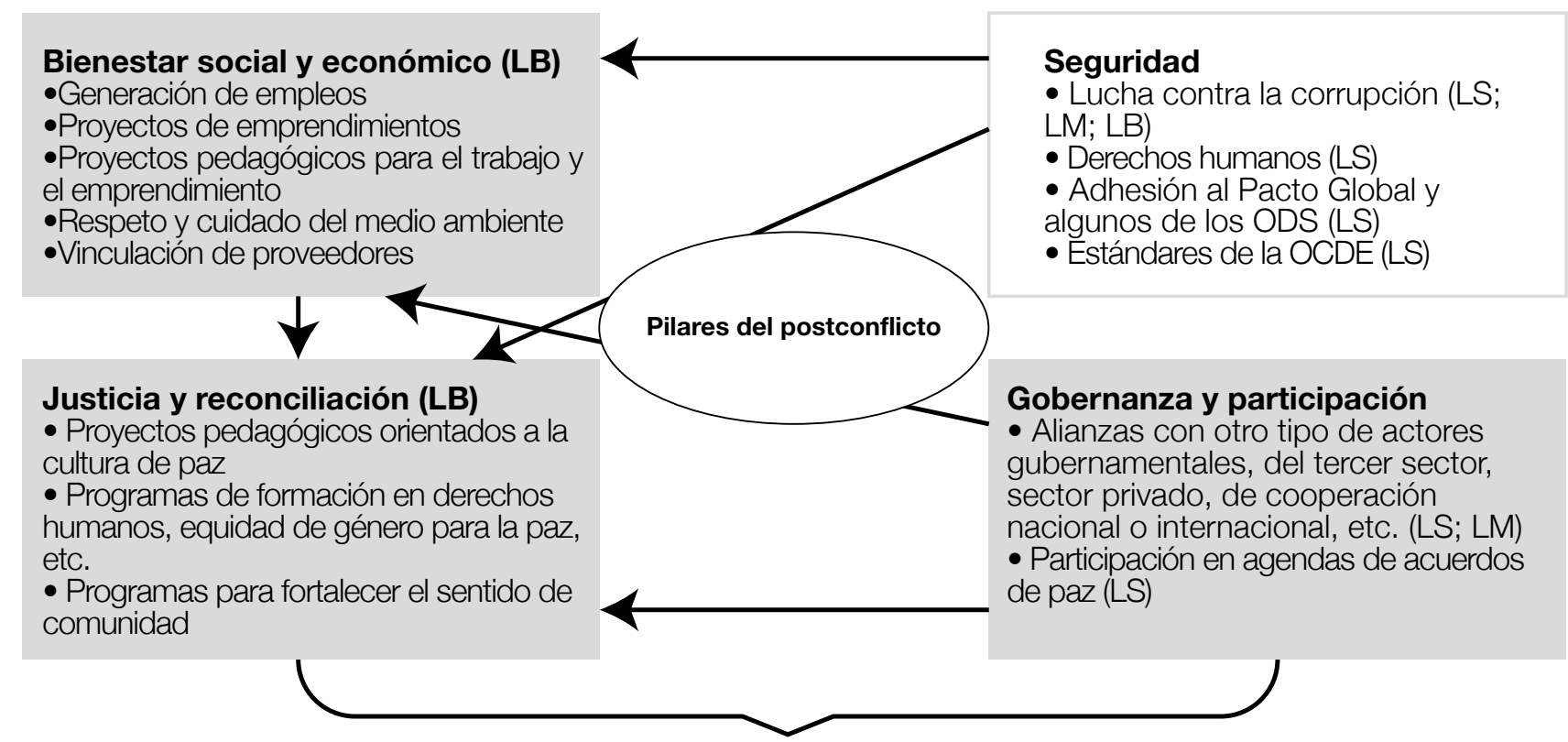

Grupos de interés en el postconflicto: víctimas, desmovilizados o excombatientes; minorías discriminadas; miembros de las fuerzas armadas y empresas de seguridad; medio ambiente; gobierno, organizaciones aliadas (públicas, privadas, del tercer sector, internacional, etc.); y la sociedad.

$\mathrm{LS}=$ Liderazgo superior

LM= Liderazgo de rango medio

$\mathrm{LB}=$ Liderazgo de base

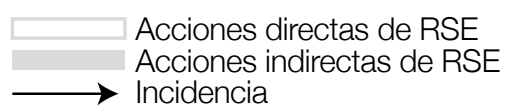

Figura 2. Prácticas de RSE orientadas a la construcción de paz en el postconflicto Fuente: elaboración propia 
Es importante resaltar que, si bien las prácticas de RSE se pueden orientar a pilares específicos del postconflicto, también pueden tener incidencia en otros, como por ejemplo cuando la generación de empleos y la vinculación de proveedores del pilar de bienestar social y económico incide en los procesos de reparación y reconciliación del pilar de justicia y reparación, o cuando la adhesión al Pacto Global y los ODS del pilar de seguridad inciden en las políticas de generación de empleo (e.g. trabajo decente, reducción de desigualdades) del pilar de bienestar social y económico (ver figura 2). Finalmente, estas prácticas de RSE se pueden orientar a uno o más de los grupos de interés que fundamentan el contexto de postconflicto, aunque como aspiración final se encuentre la construcción de paz.

\section{Conclusiones}

El objetivo del presente artículo fue analizar cuáles son las prácticas de RSE orientadas a la construcción de paz en contextos de postconflicto. Mediante una revisión de la literatura se logró evidenciar que las empresas se articulan con los objetivos de construcción de paz a través de acciones directas e indirectas de RSE que se asocian a pilares del postconflicto como el bienestar social y económico, la justicia y reconciliación, la seguridad, gobernanza y participación. Como punto de partida, se tuvo en cuenta que la construcción de paz en los escenarios de postconflicto está mediada por la noción de peacekeeping o mantenimiento de la paz, bajo el cual se busca la prevención de recaer en el conflicto y actuar sobre los efectos que este ha dejado en las sociedades. La RSE como enfoque de gestión reconoce la importancia de reflexionar sobre el papel que desempeñan las empresas en la sociedad, especialmente en lo relativo al modo en que generan sus ganancias y los impactos económicos, sociales y ambientes derivados.

Así mismo, la construcción de paz desde la RSE sugiere crear una situación de gana-gana en la que las empresas generan valor para sus grupos de interés, pero al tiempo, se benefician de la paz, que es buena para los negocios, y las consecuencias del conflicto los afectan. Esto implica reconocer que la identificación de grupos de interés y la generación de valor para ellos enmarca las prácticas de RSE para la construcción de paz en las teorías integrativas (Garriga y Melé, 2004). En el postconflicto los grupos de interés adquieren una configuración específica, a partir de su historia y relación con el conflicto. Es por ello que las investigaciones registran grupos de interés tales como víctimas (individuales y colectivas), desmovilizados o excombatientes; minorías discriminadas; miembros de las fuerzas armadas y empresas de seguridad; medio ambiente; gobierno, organizaciones aliadas (públicas, privadas, del tercer sector, internacional, etc.); y la sociedad, teniendo en cuenta además, que estos grupos de interés se deben identificar a la luz de variables como edad, género, raza, etnia, ubicación geográfica, etc., que determinan la necesidad de poner en práctica RSE específicas y un particular relacionamiento con dichos grupos.

Bajo esta dimensión teórica de la RSE orientada a la construcción de paz, se pudieron identificar en las investigaciones, prácticas enmarcadas en el pilar del postconflicto de bienestar social y económico, a través de la generación de empleos, apoyo a los emprendimientos (especialmente las actividades locales) y formación para el trabajo y los negocios. Si bien estas prácticas tienen como base el reconocimiento de las condiciones de pobreza y vulnerabilidad de algunos de sus grupos de interés, también constituyen procesos de atención y reparación de víctimas, y de reconciliación para desmovilizados o excombatientes, que por su participación en el conflicto, son marginalizados y excluidos socialmente; lo que incide en el pilar de justicia y reconciliación del postconflicto. Para las empresas estas prácticas tienen efectos en el mediano plazo, pues algunos de sus grupos de interés se pueden vincular como trabajadores, proveedores, y consumidores. 
La vinculación de estos actores del postconflicto a las empresas requiere de algunos procesos propios de la gestión humana, como las políticas del área, el análisis y diseño de cargos, los procesos de reclutamiento y selección, y la capacitación y entrenamiento, que deben tener en cuenta las características de sus habilidades y conocimientos para el trabajo. De acuerdo con las investigaciones, estos actores presentan déficit en sus perfiles para el trabajo en comparación con otro tipo de poblaciones (e.g. poblaciones urbanas, o que no se vieron afectadas por el conflicto), su adecuación puede también variar a partir de segmentos como mujeres y jóvenes, adultos mayores o grupos minoritarios. Para ello, son importantes las políticas de equidad y de oportunidades, así como la promoción de valores para aceptar a actores como los desmovilizados y excombatientes, los cuales son vistos en la sociedad como los victimarios del conflicto.

En el marco del pilar de justicia y reconciliación del postconflicto, en las investigaciones se evidenciaron prácticas como los proyectos pedagógicos o programas orientados a la promoción de los derechos humanos, equidad de género, formación para la paz, y actividades de reconciliación. Según estas investigaciones, este tipo de prácticas promueven una "cultura de paz" y procesos de reconciliación y perdón por los daños que actores como los desmovilizados o excombatientes generaron en el contexto de los conflictos, los cuales están marcados por estigmas sociales y barreras de aceptación, presentan dificultad y afrontan procesos complejos para incorporarse a la vida civil. Estas prácticas refuerzan la convivencia social y contribuyen a fortalecer el sentido de comunidad.

Las investigaciones analizadas también reconocen que las empresas no deben actuar de manera aislada en los contextos de postconflicto, sino que deberían articularse con otros actores del sector privado, público, del tercer sector y otros organismos internacionales, para construir paz de manera conjunta. Esto permite que con el aporte de las capacidades (e.g. financieras, de conocimiento, de infraestructura, políticas, experiencia, etc.) de cada actor, se logren resultados más significativos en los procesos de construcción de paz. Además, las investigaciones consideran pertinente la vinculación con las agendas de acuerdos de paz, que constituyen rutas o guías de múltiples actores para la construcción de paz. De acuerdo con lo anterior, las alianzas permiten que las empresas de la RSE se incorporen al pilar de gobernanza y participación del postconflicto.

Otras prácticas orientadas a la construcción de paz desde la RSE registradas en las investigaciones, son aquellas en las que las empresas asumen roles indirectos y se enmarcan en las teorías éticas de la RSE (Garriga y Melé, 2004). La RSE como actuar estratégico de las empresas que involucra elementos éticos, es un compromiso para el desarrollo de la sociedad en general, por lo que se orienta a las dimensiones sociales, económicas y ambientales. Desde esta perspectiva teórica, las empresas no requieren modificar sus actividades misionales, reestructurar su gobierno corporativo o crear programas de RSE para la paz, sino que de manera voluntaria, pueden tomar consciencia del modo en que su actuar ético incide en la construcción de paz.

Según las investigaciones, el respeto y la promoción de los derechos humanos, la adhesión al Pacto Global y el alcance de los ODS, las iniciativas de reportes, los códigos de ética, entre otros, permiten evidenciar transparencia y un actuar ético ante la sociedad, así como rechazar la corrupción y las actividades ilegales, elementos que abarcan el pilar de seguridad del postconflicto. Adicional a ello, las investigaciones reconocen que este tipo de prácticas inciden en otros pilares como el bienestar social y económico, la justicia y reconciliación, puesto que no solo abarcan los derechos humanos, sino también principios como el trabajo decente, el crecimiento económico, la disminución de desigualdades, la equidad, la paz y la justicia, entre otros. 
Bajo la adaptación de Killick de la pirámide de Lederach sobre los actores y enfoques para construcción de paz en los negocios (Nelson, 2000), se argumentó que las empresas pueden emprender prácticas de RSE orientadas a la paz, a partir de los roles de liderazgo superior, medio y de base. Esto permite comprender que la construcción de paz desde la RSE es posible desde cualquier nivel de autoridad en las empresas y a través de prácticas directas e indirectas.

Uno de los debates que plantean algunas investigaciones como las de Boudreaux (2007), Buitrago et al. (2015), McKenna (2014; 2016), Prandi y Lozano (2010), Tobias y Boudreaux (2011), estriban en la escasa atención que se ha prestado al medioambiente en los estudios de RSE y construcción de paz. Para las nombradas investigaciones, esto ha servido de escenario para los actos bélicos y la afectación del medioambiente, por lo cual es necesario reconciliarse con él, repararlo y generar conciencia de la importancia de su protección.

Para futuras investigaciones se recomienda analizar las dimensiones discutidas en el presente artículo en contextos específicos, como es el caso colombiano, para identificar hasta qué punto las empresas han desarrollado prácticas de RSE para responder a los retos del postconflicto y la construcción de paz. Así mismo, la discusión desarrollada en este artículo es una reflexión para visibilizar que existen diversas apuestas que pueden implementar las empresas, independiente de su misión, tamaño y del sector al que pertenezcan, las cuales pueden ser directas o indirectas, desarrollarse desde distintos niveles jerárquicos y orientarse a diferentes grupos de interés.

\section{Referencias}

Abramov, I. (2009). Building peace in fragile States building trust is essential for effective public-private partnerships. Journal of Business Ethics, 89, 481-494. https://doi.org/10.1007/s10551-010-0402-8
Allan, G. (2001). Pace Building: The Private Sector's Role. American Journal of International Law, 95(1), 102-119.

Baek, P., y Kim, N. (2014). Exploring a theoretical foundation for HRD in society: toward a model of stakeholderbased HRD. Human Resource Development International, 17(5), 499-513. https://doi.org/10.108 0/13678868.2014.954189

Banks, H. (2016). The business of peace: Coca-Cola's contribution to stability, growth, and optimism. Business Horizons, 59(5), 455-461. https://doi. org/10.1016/j.bushor.2016.03.018

Barnett, M., Kim, H., O'Donnell, M., y Sitea, L. (2007). Peacebuilding: What is in a Name? Global Governance, 13, 35-58.

Barrios, R. (2016). Responsabilidad social empresarial: el papel de las organizaciones en el postconflicto colombiano. Ensayos, 9(1), 45-56. https://revistas.unal.edu.co/ index.php/ensayos/article/view/61276/57712

Bedoya, C. (2017). El papel de las empresas en la construcción de la paz en Colombia: un análisis en clave simétrico. En Encuentro Internacional de Investigadores en Administración 2017. Universidad Externado de Colombia; Universidad del Valle.

Bedoya, C., y Charfuelán, M. (2019a). Las empresas y la construcción de paz: una revisión sistemática en la literatura. En Paz y Diversidad: Una mirada desde la academia (pp.107-128). Sello Editorial Unicatólica.

Bedoya, C., y Charfuelán, M. (2019b). Acciones e iniciativas de paz de organizaciones en el Valle del Cauca: experiencias desde el sector público, privado y tercer sector. Sello Editorial Unicatólica.

Bennett, J. (2002). Multinational Corporations, Social Responsibility and Conflict. Journal of International Affairs, 55(2), 393 410. https://pdfs.semanticscholar.org/5336/c0274287e44 3033c60fa25e143150b44154d.pdf

Bond, C. J. (2014). Business, Peace and Mining: A Literature Review. Business, Peace and Sustainable Development, 4, 59-83. https://doi.org/10.9774/GLEAF.8757.2014. no.00005

Boudreaux, K. (2007). The business of reconciliation: Entrepreneurship and commercial activity in postconflict Rwanda. Economic Affairs, 27(2), 6-13. https://doi.org/10.1111/j.1468-0270.2007.00724.x 
Boutros-Ghali, B. (1992). An Agenda for Peace: Preventive Diplomacy, Peacemaking and Peace-keeping. Report of the Secretary-General. Organización de las Naciones Unidas. https://doi. org/10.1177/004711789201100302

Buitrago, N., Bustamante, A., Castañeda, S., y Ramírez, S. (2015). Retos de las organizaciones privadas en el postconflicto colombiano. Revista Ensayos, 8, 228-239.

Carroll, A. B. (1999). Corporate Social Responsability. Evolution of a Definitional Construct. Business \& Society, 38(3), 268-295.

Carroll, A.B., y Shabana, K.M. (2010). The Business Case for Corporate Social Responsibility: A review of concepts, research and practice. International Journal of Management Reviews, 12, 85-105.

Chowdhury, M. J. A. (2011). The Determinants of Entrepreneurship in a Conflict Region: Evidence from the Chittagong Hill Tracts in Bangladesh. Journal of Small Business E Entrepreneurship, 24(2), 265-281. https://doi.org/10.1080/08276331.2011.10593538

Dahlsrud, A. (2008). How Corporate Social Responsibility is Defined: an Analysis of 37 Definitions. Corporate Social Responsibility and Environmental Management, 15, 1-13.

De-San-Eugenio, J., Ginesta, X., y Xifra, J. (2017). Peace, sports diplomacy and corporate social responsibility: a case study of Football Club Barcelona Peace Tour 2013. Soccer and Society, 18(7), 836-848. https://doi.org/10.1080/1466097 0.2015 .1067796

Dentchev, N. A. (2004). Corporate Social Performance as a Business Strategy. Journal of Business Ethics, 55(4), 395-410.

Flavin, W. (2003). Planning for conflict termination and postconflict success. Parameters, 33(3), 95-112.

Ford, J. (2015). Perspectives on the Evolving "Business and Peace" Debate. Academy of Management Perspectives, 29(4), 451-460. https://doi.org/10.5465/ amp. 2015.0142

Forrer, J., Fort, T. y Gilpin, R. (2012). How Business Can Foster Peace. Special Report. Usip, Washington, DC.

Forrer, J. J., y Katsos, J. E. (2015). Business and Peace in the Buffer Condition. Academy of Management Perspectives, 29(4), 438-450. https://doi.org/10.5465/amp.2013.0130
Fort, T.L. (2007). Business, Integrity, and Peace. Cambridge University Press.

Fort, T. L., y Schipani, C. A. (2002). The Role of the Corporation in Fostering Sustainable Peace. Vanderlit Journal of Transnational Law, 35(2), 389-436. https:// doi.org/10.2139/ssrn.303316

Fort, T. L., y Schipani, C. A. (2007). An action plan for the role of business in fostering peace. American Business Law Journal, 44(2), 359-377. https://doi. org/10.1111/j.1744-1714.2007.00040.x

Freeman, R. E. (1984). Strategic management: A stakeholder approach. Pitman.

Fuentes-García, F. J., Núñez-Tabales, J. M., y Veroz-Herradón, R. (2008). Applicability of Corporate Social Responsibility to Human Resources Management: Perspective from Spain. Journal of Business Ethics, 82(1), 27-44. https:// doi.org/10.1007/s10551-007-9560-8

Gallego, L., Guitiérrez, I., Osorio, D., y Cortés, A. (2016). Los retos de los empresarios en la construcción de paz. Universidad Eafit.

García, J., Durán, S. E., Parra, M. A., y Martínez, H. (2018). Inserción, integración y equidad en el ámbito laboral: escenario empresarial posconflicto en Colombia. Revista de Ciencias Sociales, 24(3), 36-49.

Garriga, E., y Melé, D. (2004). Corporate Social Responsibility Theories: Mapping the Territory. Journal of Business Ethics, 53, 51-71.

Gerson, A. (2001). Peace Building: The Private Sector's Role. The American Journal of International Law, 95(1), 102-119.

Ghimire, S., y Raj Upreti, B. (2012). Corporate Engagement for Conflict Transformation: Conceptualising the Business-Peace Interface. Journal of Conflict Transformation E Security, 2(1), 77-100.

Gobierno Nacional de Colombia, Farc-EP (2016). Acuerdo Final para la Terminación del Conflicto y la Construcción de una Paz Estable y Duradera. http:// www.altocomisionadoparalapaz.gov.co/procesos-yconversaciones/Documentos\%20compartidos/24-112016NuevoAcuerdoFinal.pdf

González, J. (2016). Empresa privada: principal socio en el posconflicto y la construcción de paz. Panorama, 10(18), 84-92. 
Grasa, R., y Mateos, O. (2014). Guía para trabajar en la construcción de la paz. qué es y qué supone la construcción de la paz. Cámara de Comercio de Bogotá; Instituto Catalán Internacional para la Paz.

Hagerty, T. (2013). The Butcher, the Baker, the Brewer and the Peacebuilder. Oportunities, Strategies and Metrics for the Role of Business in Building Peace. Business, Peace and Sustainable Development, 1, 69-78.

Hamre, J. J., y Sullivan, G. R. (2002). Toward Postconflict Reconstruction. Washington Quarterly, 25(4), 85-96. https://doi.org/10.1162/016366002760252554

Hayhurst, L. M. C., y Szto, C. (2016). Corporatizating Activism Through Sport-Focused Social Justice? Investigating Nike's Corporate Responsibility Initiatives in Sport for Development and Peace. Journal of Sport and Social Issues, 40(6), 522-544. https://doi.org/10.1177/0193723516655579

Hönke, J. (2014). Business for peace? The ambiguous role of 'ethical' mining companies. Peacebuilding, 2(2), 172-187. https://doi.org/10.1080/21647259.2014.910383

Ibisate, Á. (2008). La relación con los grupos de interés. En A. Catalunya (ed.), La Aplicación de la Responsabilidad Social a la Gestión de las Personas (pp. 127-132). Media Responsable.

Jiménez-Bautista, F. (2012). Conocer para comprender la violencia: origen, causas y realidad. Convergencia Revista de Ciencias Sociales, 19(58), 13-52.

Jiménez-Peña, G. (2014). Multinacionales y responsabilidad social empresarial en la construcción de paz en Colombia. Cuadernos de Administración, 27(48), 6796.

Kanashiro, P., y Starik, M. (2016). Business efforts, opportunities, and limits addressing the poor: A Brazilian case study. Business Horizons, 59(5), 471-479. https://doi.org/10.1016/j. bushor.2016.03.011

Katsos, J. E., y Forrer, J. (2014). Business practices and peace in post-conflict zones: Lessons from Cyprus. Business Ethics, 23(2), 154-168. https://doi.org/10.1111/ beer. 12044

Kolk, A., y Lenfant, F. (2013). Multinationals, CSR and Partnerships in Central African Conflict Countries. Corporate Social Responsibility and Environmental
Management, 20(1), 43-54. https://doi.org/10.1002/ csr. 1277

Kytle, B., y Ruggie, J. G.(2005). Corporate Social Responsibility as Risk Management: A model for multinationals. Corporate Social Responsibility Initiative Working Paper. Cambridge, MA.: John F. Kenndy School of Government, Harvard University.

Ledbetter, B. (2016). Business leadership for peace. International Journal of Public Leadership, 12(3), 239. 251. https://doi.org/10.1108/IJPL-04-2016-0016

Maconachie, R. (2016). The extractive industries, mineral sector reform and post-conflict reconstruction in developing countries. The Extractive Industries and Society, 3(2), 313-315. https://doi.org/10.1016/j. exis.2016.03.004

McKenna, K. (2014). Business and Peace. Lesson from Bougainville. Business, Peace and Sustainable, 2, 28-55.

McKenna, K. (2016). Land of the Unexpected Natural Resource Conflict and Peace Building in Papua New Guinea. Business, Peace and Sustainable Development, 7, 32-49. https://doi.org/10.9774/ GLEAF.8757.2016.ju.00004

McNulty, R. E. (2014). The Global Compact's Inaugural Business for Peace Annual Event. Business, 5, 63-74.

Melo, J., Sánchez, K., y García, M. (2011). Responsabilidad Social Empresarial desde la Gestión Humana: Guía de primeros pasos. Programa Editorial Universidad del Valle.

Miklian, J., y Rettberg, A. (2017). From War-Torn to PeaceTorn? Mapping Business Strategies in Transition from Conflict to Peace in Colombia. SSRN Electronic Journal, 1-25. https://doi.org/10.2139/ssrn.2925244

Molina, N. (2004). Resistencia Comunitaria y transformación de Conflictos. Un análisis desde el conflicto Político Armado de Colombia. Universidad Autónoma de Barcelona.

Montinola, G. R., y Jackman, R. W. (2002). Sources of corruption: A cross-country study. British Journal of Political Science, 32(01), 147-170. https://doi. org/10.1017/S0007123402000066

Nelson, J. (2000). The Business of Peace. The private sector as a partner in conflict prevention and resolution. International Alert, Council on Economic Priorities, The Prince of Wales Business Leaders Forum. 
Noland, J., y Phillips, R. (2010). Stakeholder Engagement, Discourse Ethics and Strategic Management. International Journal of Management Reviews, 12(1), 39-49. https://doi. org/10.1111/j.1468-2370.2009.00279.x

Oetzel, J., y Breslauer, M. (2015). Editorial: The Business and Economics of Peace: Moving the Agenda Forward. Business, Peace and Sustainable, (6), 3-8. https://doi. org/10.9774/GLEAF.8757.2015.de.00002

Oetzel, J., Getz, K. a., y Ladek, S. (2007). The role of multinational enterprises in responding to violent conflict: A conceptual model and framework for research. American Business Law Journal, 44(2), 331-358. https:// doi.org/10.1111/j.1744-1714.2007.00039.x

Oetzel, J., Westermann-behaylo, M., Koerber, C., Timothy, L., y Rivera, J. (2009). Business and Peace: Sketching the Terrain. Journal of Business Ethics, 89, 351-373.

Organización Internacional de Normalización. (2014). ISO 26000. Guía sobre responsabilidad social. Ginebra.

Penh, B. (2009). New convergences in poverty reduction, conflict, and state fragility: What business should know. Journal of Business Ethics, 89, 515-528. https:// doi.org/10.1007/s10551-010-0404-6

Porter, M. E., y Kramer, M. R. (2002). The competitive advantage of corporate philanthropoy. Harvard Business Review, 80(12), 56-68.

Porter, M. E., y Kramer, M. R. (2011). La creación de valor compartido. Harvard Business Review. América Latina, 3-18.

Prandi, M. (2010). Del conflicto armado a la construcción de paz. en M. Prandi y J. M. Lozano (eds.), La RSE en contextos de conflicto y postconflicto: de la gestión del riesgo a la creación de valor (pp. 17-33). Escola de Cultura School for a Culture of Peace (UAB), Institute for Social Innovation (Esade).

Prandi, M., y Lozano, J. M. (eds.). (2010). La RSE en contextos de conflicto y postconflicto: de la gestión del riesgo a la creación de valor. Escola de Cultura de Pau (UAB), Instituto de Innovación Social (Esade).

Rahman, S. (2011). Evaluation of Definitions: Ten Dimensions of Corporate Social Responsibility. World Review of Business Research, 1(1), 166-176.
Ramnarain, S. (2015). Interrogating women's peace work: Community-based peacebuilding, gender, and savings' co-operatives in post-conflict Nepal. Community Development Journal, 50(4), 677-692. https://doi.org/10.1093/cdj/bsu065

Rathmell, A. (2005). Planning post-conflict reconstruction in Iraq: what can we learn? International Affairs, 81(5), 1013-1038.

Raufflet, E. (2010). Responsabilidad corporativa y desarrollo sostenible: una perspectiva histórica y conceptual. Cuaderno de Administración, (43), 23-32.

Rettberg, A. (2002). Administrando la adversidad: respuestas empresariales al conflicto colombiano. Colombia Internacional, (55), 37-54.

Rettberg, A. (2007). The private sector and peace in El Salvador, Guatemala, and Colombia. Journal of Latin American Studies, 39, 463-494.

Rettberg, A., Camacho, A., Chaux, E., García, A., Iturralde, M., Sánchez, F., .. Wills, L. (2002). Preparar el futuro: conflicto y post-conflicto en Colombia. Universidad de los Andes; Fundación Ideas para la Paz; Alfaomega.

Rettberg, A., Leiteritz, R., y Nasi, C. (2011). Entrepreneurial Activity in the Context of Violent Conflict: Business and Organized Violence in Colombia. Journal of Small Business \& Entrepreneurship, 24(2), 37-41. https://doi.org/10.1080/08276331.2011.10593533

Rettberg, A., y Rivas, A. (2012). El sector empresarial y la Construcción de paz en Colombia: entre el optimismo y el desencanto. En Construcción de Paz en Colombia (pp. 305-348). Ediciones Uniandes.

Rodríguez, A. L., y Gamboa, R. (2017). Gestión del Postconflicto como estrategia de la Responsabilidad Social en las empresas. Revista de La Facultad de Ciencias Económicas y Empresariales, 17(1), 35-44.

Rolston, B. (2007). Demobilization and reintegration of Ex-combatants: The irish case in international perspective. Social and Legal Studies, 16(2), 259-280. https://doi.org/10.1177/0964663907076534

Ruiz, J. (2003). Administrando la adversidad: respuestas empresariales al conflicto Colombiano. Colombia Internacional, 55, 37-54. 
Ruiz, M. I., Galeano, J. S., y Gil, E. O. (2017). Posconflicto colombiano. Revista CIFE: Lecturas de Economía Social, 17(27), 23-54.

Ryan, J. (2012). Infrastructures for Peace as a Path to Resilient Societies: An Institutional Perspective. Journal of Peacebuilding \& Development, 7(3), 14-24.

Sepúlveda, M. E., Moreno, E. P., Tovar, J. E., Franco, J. A., y Villarraga, C. L. (2015). Responsabilidad social de las PyMEs en el marco del posconflicto, como soporte para la inclusión laboral de la población reinsertada. Revista de Ciencias Sociales, 6(1), 45-62.

Tobias, J. M., y Boudreaux, K. C. (2011). Entrepreneurship and Conflict Reduction in the Post-Genocide Rwandan Coffee Industry. Journal of Small Business \& Entrepreneurship, 24(2), 217-242. https://doi.org/10. 1080/08276331.2011.10593535

Ugarriza, J. E. (2013). La dimensión política del postconflict : discusiones conceptuales y avances empíricos. Colombia Internacional, (77), 141-176.

World Bank. (1995). The International Bank for Reconstruction and Development. Washington: World Bank.

van Dorp, M. (2017). Should companies be involved in peacemaking, or mind their own business? Global Change, Peace and Security, 29(1), 95-102. https:// doi.org/10.1080/14781158.2016.1256278

van Marrewijk, M. (2003). Concepts and Definitions of CSR and Corporate Sustainability: Between Agency and Communion. Journal of Business Ethics, 44(2-3), 95-105.
Vargas, J. (2012). Costos del conflicto y consideraciones económicas. En A. Rettberg (ed.), Construcción de Paz en Colombia (pp. 239-272). Ediciones Uniandes.

Voegtlin, C., y Greenwood, M. (2016). Corporate social responsibility and human resource management: A systematic review and conceptual analysis. Human Resource Management Review, 26, 181-197. https:// doi.org/10.1016/j.hrmr.2015.12.003

Wielandt, G. (2005). Hacia la construcción de lecciones del posconflicto en América Latina y el Caribe: una mirada a la violencia juvenil en Centroamérica. Politicas Sociales N. ${ }^{\circ} 115$. Cepal.

Wilcox, T. (2006). Human resource development as an element of corporate social responsibility. Asia Pacific Journal of Human Resources, 44(2), 184-196. https://doi.org/10.1177/1038411106066395

Wilson, S. A. (2015). Corporate social responsibility and power relations: Impediments to community development in post-war Sierra Leone diamond and rutile mining areas. Extractive Industries and Society, 2(4), 704-713. https:// doi.org/10.1016/j.exis.2015.09.002

Yoosuf, A., y Premaratne, S. P. (2017). Building Sustainable Peace through Business Linkages among MicroEntrepreneurs: Case Studies of Micro-Enterprises in the North of Sri Lanka. Journal of Peacebuilding and Development, 12(1), 34-48. https://doi.org/10.1080/ 15423166.2017.1281754 